\title{
A Refined Concentric Anchor-Beacons Location Algorithm for Wireless Sensor Networks Based on Received Signal Strength Indicator
}

\author{
Zhang Kun \\ 72465 Unit of PLA \\ Jinan, China \\ e-mail:zkun8@163.com \\ Chen $\mathrm{He}$ \\ 72465 Unit of PLA \\ Jinan, China \\ e-mail:chenhe@163.com
}

\author{
Zhang Can \\ China University of Mining and Technology \\ Xuzhou, China \\ 408111448@qq.com \\ Yin Xiaohu \\ 72465 Unit of PLA \\ Jinan, China \\ 646708564@qq.com
}

\begin{abstract}
As the development of technology, the wireless sensor networks (WSN) have a wide spread usage. And people pay more attention on the localization algorithm, as the key technology of WSN, there have been many method of selflocalization. The concentric anchor-beacons (CAB) location algorithm is one of the most practical one, which is a rangefree WSN localization algorithm. In order to further improve the accuracy of localizing nodes, an improved CAB location algorithm base on Received Signal Strength Indicator (RSSI) is proposed. The RSSI is used to measure the distance between two anchors and compare with the practical distance. Then the environment between two anchors can be simulated. At last the communication radius of anchors can be optimized. And the common area of the anchors in the process of localizing nodes can be reduced. Then the accuracy is improved. By simulation, the localization accuracy is improved when the anchors numbers is more than a certain percentage.
\end{abstract}

Keywords-Wireless sensor networks; received signal strength indicator; concentric anchor-beacons; node localization

\section{INTRODUCTION}

Wireless Sensor Networks (WSN) are the self-organizing networks that are constituted by a large number of embedded sensor nodes arranged in the monitoring region ${ }^{[1]}$.

Depending on the characteristics of low cost, easy layout and flexible network structure, WSN have been applied extensively in many areas such as national defense and military, environmental monitoring, disaster relief, medical treatment and public health and the agriculture and industrial controls. Sensor nodes in the networks distributed in the area monitor and collect the local temperature and humidity, illumination intensity, vehicles or personnel information and its movement, and deliver them to the monitors by the networks ${ }^{[2]}$. However, there is no sense to monitors that only know this information. The key to monitors is to know the location where the information is sent, which is achieved by the nodes localization technology of WSN. Therefore, the nodes localization is an important part of WSN, and is an important aspect of network research.
There are many methods to locate nodes. According to the need of measure the distance between the unknown nodes and anchor node, location algorithm can be divided to ranging and no ranging ${ }^{[3]}$. As it dosen't need additional hardware supports, lower power consumption and cost, and a simple calculation, the latter has more research value than the former. But Concentric Anchor Beacons (CAB) location algorithm is a more practical method in no ranging currently. There are many scholars have put forward their own suggestions for the improvement of CAB location algorithm. Jiang Zhipeng et al. ${ }^{[4]}$ have proposed a way of improving localizing accuracy by using unknown nodes in adjoining circulars communicating with each other to reduce the crossarea, but this way increases energy cost of unknown nodes, and is not conducive to the long-term work. Li Yaoyao et al.

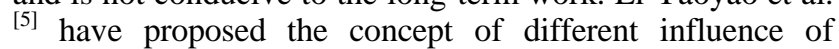
anchors and using the method of weighting, thereby improved the accuracy of the algorithm of the concentric circles. However, they only replaced it with model and haven't analyzed the reasons of different influence and specific influential factors completely.

All of the anchors in the $\mathrm{CAB}$ location algorithm are provided with the same radius, so as to be the same energetic classification. But in applications, due to the influence of the external environment, every communicating radius of the anchors is not the same. How to determine a communicating radius that adapt to environment for anchors, thereby reducing the error of unknown nodes localization, this paper presents a scheme that optimized information of anchors base on RSSI(Received Signal Strength Indicator), and improves localization accuracy of CAB location algorithm on .

\section{The PRINCIPLE OF CAB LOCATION ALgORITHM AND RSSI}

\section{A. The Principle of $C A B$ location Algorithm}

Anchor is the center of $\mathrm{CAB}$ and the radius equal to a number of wireless communication ranges. Shah et al. ${ }^{[6]}$ have confirmed that compared with center of mass algorithm, $\mathrm{CAB}$ can greatly reduce errors of node localization when 
system selected two power levels to locate, but its localizing performance is not enhanced when system selects three power levels further. Therefore, this paper studies the CAB based on only two classifications, and takes $1: \sqrt{2}$ as the radius relationship between two energy levels.

In the $\mathrm{CAB}$, in order to locate accurately, the unknown nodes must receive broadcast information from three anchors at least. As shown in Figure 1, it is assumed that the unknown nodes can receive the information from three beacon nodes, and the unknown nodes locate on the internal intersection region, then the unknown nodes position can be got by intersection point coordinates.

It is assumed that the coordinates of three anchors S1, S2, S3 are $\left({ }^{x_{s_{1}}}, y_{s_{1}}\right),\left({ }^{x_{s_{2}}}, y_{s_{2}}\right),\left({ }^{x_{s_{3}}, y_{s_{3}}}\right)$, the communication radius of nodes is $\mathrm{R}$, then the communication radius of anchors of the first energy level is $\sqrt{2} / 2 R$. All of intersection of concentric circles can be got according to following solves (1):

$$
\left\{\begin{array}{l}
\left(x-x_{s_{1}}\right)^{2}+\left(y-y_{s_{1}}\right)^{2}=(\sqrt{2} / 2 R)^{2} \\
\left(x-x_{s_{1}}\right)^{2}+\left(y-y_{s_{1}}\right)^{2}=R^{2} \\
\left(x-x_{s_{2}}\right)^{2}+\left(y-y_{s_{2}}\right)^{2}=(\sqrt{2} / 2 R)^{2} \\
\left(x-x_{s_{2}}\right)^{2}+\left(y-y_{s_{2}}\right)^{2}=R^{2} \\
\left(x-x_{s_{3}}\right)^{2}+\left(y-y_{s_{3}}\right)^{2}=(\sqrt{2} / 2 R)^{2} \\
\left(x-x_{s_{3}}\right)^{2}+\left(y-y_{s_{3}}\right)^{2}=R^{2}
\end{array} .\right.
$$

Meanwhile, the coordinates that compose of the intersection of the public areas in (1) satisfy the relationship (2):

$$
\left\{\begin{array}{l}
(\sqrt{2} / 2 R)^{2} \leq\left(x-x_{s_{1}}\right)^{2}+\left(y-y_{s_{1}}\right)^{2} \leq R^{2} \\
(\sqrt{2} / 2 R)^{2} \leq\left(x-x_{s_{2}}\right)^{2}+\left(y-y_{s_{2}}\right)^{2} \leq R^{2} \\
(\sqrt{2} / 2 R)^{2} \leq\left(x-x_{s_{3}}\right)^{2}+\left(y-y_{s_{3}}\right)^{2} \leq R^{2}
\end{array}\right.
$$

Figure 1. A diagram of $\mathrm{CAB}$ localization.

The coordinates of unknown nodes can be obtained by the intersection of the average after calculating intersection coordinates.

\section{B. The Principle of RSSI}

RSSI is given transmit power, and measured received power at the receiving nodes to calculate propagation loss, then converted the propagation loss into distance by using of the theory or experience signal propagation model. It uses of RF signal mainly. As it is with wireless communication capability, sensor nodes are low power and inexpensive ranging technology. In RSSI, nodes compare signal power which receives by itself to transmit power, and convert power variations to distance data based on the corresponding signal propagation model.

The calculation method is:

$\mathrm{RSSI}=$ transmit power + antenna gain - path loss.

\section{OPTIMIZATION PRINCIPLE}

The premise of CAB is assuming that nodes have ideal range of circular wireless signal propagation, therefore the communication range of each anchors are circles with the same radius in the algorithm, but it can't be achieved at practical environment, as environments is different for each anchor in application process, communication radius will change irregularly in every direction under the influence of the surroundings, thereby resulting the range of all anchors being randomization and divergence. The change can't be obtained by a simple method, but if the change can be expressed by a simple operation, the unknown nodes localization will be more accurate.

According to the principle of RSSI, we can obtain the distance between the adjacent anchors which be referred as test distance. And since the position of the anchors in the field is known, the distance between the anchors can be obtained by a simple calculation which called actual distance. According to the difference of environmental impact, difference exists between test distance and actual distance, and the reason leads to this is external influencing factors which cause change of anchors communication range.

Because the change of anchors communication range which is leaded by external influence is irregular, the difficulty of calculation is increased greatly. In this paper, we consider synthesizing the effect of each factor to take the average, and then get a new communication radius.

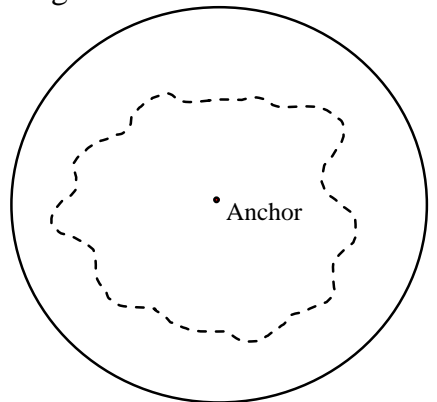

Figure 2. Ideal communication range of anchors (inside the solid line) and the actual communication range (inside the dotted line).

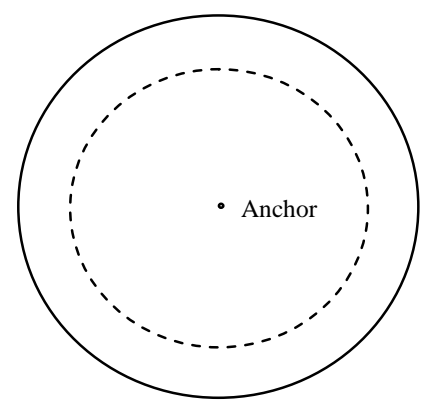

Figure 3. The ideal anchors communication range (inside the solid line) and the optimized communication range (inside the dotted line). 


\section{Algorithm flow}

1) Each anchor periodically broadcasts its location information outwardly with certain signal strength.

2) According to the received location information of the other anchors, Each anchor take the average of the RSSI of the same anchor, calculating the distance between two anchors by RSSI, and calculate actual distance between two anchors, and then get error between two anchors by comparing the two parameters above.

3) Repeat 2) to all received messages of anchors and get error among all around anchors.

4) Determine a reasonable communication radius for anchors according to the principle of minimum variance.

5) Anchors retransmit broadcast information which contains anchors position, communication radius, and energy level identification.

6) Unknown nodes choose anchors and locate.

\section{SimULATION ANALYSIS}

In this paper, we use MATLAB 7.0 to carry out simulation of nodes localization. The simulation environment is an area $(150 \mathrm{~m} \times 150 \mathrm{~m})$ in which is arranged anchors and unknown nodes at random, anchors theoretical communication radius is $20 \mathrm{~m}$, and take the average of the results 50 times as a basis for verification.

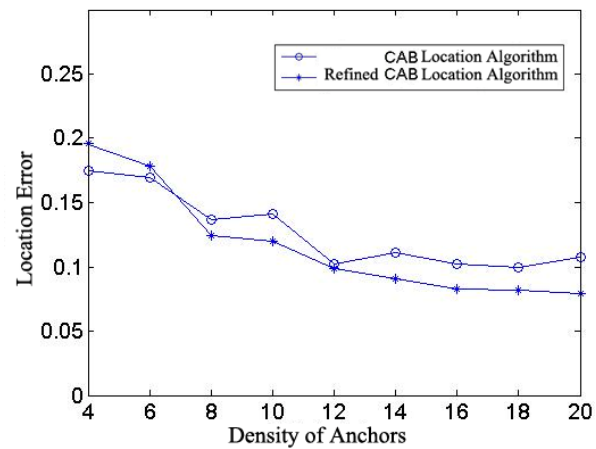

Figure 4. The comparison of localization error under different numbers of anchors.

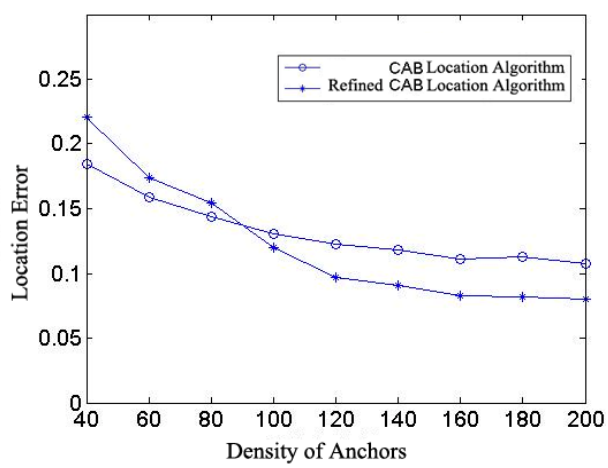

Figure 5. The comparison of localization error under different numbers of nodes.
In Fig. 4, density of anchors is $20 \%$, and corresponding localization error is under different numbers of nodes. In Fig. 5, 200 nodes are arranged in internal area, and corresponding localization error is under different numbers of anchors. As can be seen, when anchors are fewer, the improvement is not obvious, even is worse than the $\mathrm{CAB}$ algorithm, but as anchors increase, improving effect is obvious.

Analyzes the causes, when anchors are few, many unknown nodes are in the second energy level of anchors, and three rings intersect, to reduce the area of radius intersecting is not reduced necessarily, but as long as radius is reduced in the first energy level of anchors, the intersecting area is reduced necessarily,

Therefore, with the numbers of anchors increasing, anchor broadcasts that unknown nodes can receive will increase, and the distance with anchors will become nearer, and the probability of locating in the first energy level of anchors will increase greatly, and location error will reduce relative to $\mathrm{CAB}$ algorithm.

\section{CONCLUSIONS}

This paper studies the optimization of CAB location algorithm for wireless sensor networks based on RSSI. RSSI measures the communication distance between the adjacent anchors, and compares with its actual distance, and simulates communication environment around anchors, and then optimizes the actual communication radius.

By optimizing, the intersection area of each anchor of concentric circles is reduced when unknown nodes locate, thereby reduce the nodes localization errors and improve localization accuracy. The simulation results show that the algorithm can reduce localization errors of CAB location algorithm, and can satisfy better to nodes low-cost and lowpower requirements of wireless sensor networks, and it is a practical scheme for localization.

[1] I F Akyildiz, W Su, Y Sankarasubramaniam, E Cayirci. Wireless sensor networks: a survey[J]. Computer Networks, 2002, 38(4): 393422

[2] L Mills Kevin. A brief survey of self-organization in wireless sensor networks [J]. Wireless Communications and Mobile Computing (S1530-8669), 2007, 7(7): 823-834.

[3] N Shah, I Zeid. Ad hoc localization technique for wireless Sensor networks [J]. The International Society for Optical Engineering (S0277-786X), 2005, v6011:60110A1-60110A12, 2005.

[4] Jiang Zhipeng, Gao Suixiang. A Refined Concentric Anchor-Beacons Algorithm for Wireless Sensor Networks [J]. Computer Science, 2009,36 (10) : 46-54.

[5] Li Yaoyao, Liao Hongyun, Zeng Xiaoping, Wu Xiaolin. A Refined Weighted centroid localization Algorithm for Wireless Sensor Networks Based on Concentric Anchor [J].Computer Simulation, 2011,28 (6) : 141-144.

[6] V Vivekanandan, W S Vincent. Concentric anchor beacon localization algorithm for wireless sensor networks [J]. IEEE Transactions on Vehicular Technology, 2007, 56(5): 2733-2744.

[7] T S Rappaport. Wireless Communications: principles and practice [M]. Prentice Hall: New Jersey, 1996, 50-143. 